\title{
Aerosol particle molecular spectroscopy
}

\author{
S. Arnold, E. K. Murphy, and G. Sageev
}

\begin{abstract}
The molecular spectroscopy of a solution particle by structure resonance modulation spectroscopy is discussed [S. Arnold and A. B. Pluchino, "Infrared Spectrum of a Single Aerosol Particle by Photothermal Modulation of Structure Resonances," Appl. Opt. 21, 4194 (1982); S. Arnold et al., "Molecular Spectroscopy of a Single Aerosol Particle," Opt. Lett. 9, 4 (1984)]. Analytical equations are derived for time dependence of the particle radius as it interacts with a low intensity IR source $\left(<20 \mathrm{~mW} / \mathrm{cm}^{2}\right)$. This formalism is found to be in good agreement with pulsed experiments. Working equations for the spectroscopy are derived for both constant and periodic IR excitation.
\end{abstract}

\section{Introduction}

Recently a means for obtaining the IR absorption spectrum of a single aerosol particle was disclosed. This spectroscopy, ${ }^{1,2}$ based on the IR modulation of visible structure resonances, opens a new window for following the molecular nature of a solution particle in the presence of radiation and various gases. A clear application of structure resonance modulation spectroscopy (SRMS) ${ }^{2}$ is in elucidating the role of gaseous reactants in the acidification of an aerosol droplet. In addition, since SRMS depends on radiation-induced evaporation from the droplet, the spectroscopy may be used in understanding local processes involved in the propagation of IR radiation through an aerosol.

In what follows we (a) briefly review the SRMS technique, (b) disclose a simple model for explaining the modulation of structure resonances, and (c) show that this model gives good agreement with our present experimental data.

\section{Structure Resonance Modulation Spectroscopy}

$\mathrm{SRMS}^{2}$ utilizes the narrow morphological resonances of a single micron sized particle as seen in the visible as a caliper for measuring the size change of the particle in the presence of IR radiation. This technique enables one to obtain a quantitative broadband IR absorption

S. Arnold and E. K. Murphy are with Polytechnic Institute of New York, Physics Department, Brooklyn, New York 11201. The other author is with California Institute of Technology, Department of Chemical Engineering, Pasadena, California 91125.

Received 17 August 1984.

0003-6935/85/071048-06\$02.00/0.

(C) 1985 Optical Society of America. spectrum of the particle ${ }^{1,2}$ and to follow temporal effects associated with this absorption. Thus the SRMS approach is distinct from experiments on a polydispersed cloud of submicron particles, because in such a sample the structure resonance caliper is not available and polydispersivity keeps one from understanding the detailed interaction of the IR radiation with a single particle. ${ }^{1}$ Nonetheless for a solution droplet the manner in which particle size is changed in the presence of IR radiation was first proposed to understand experimental observations on a polydispersed submicron aerosol. ${ }^{3}$ Thus Campillo et al. ${ }^{3}$ have shown that the heat imparted to an aerosol cloud by a $\mathrm{CO}_{2}$ laser beam may be used to modulate the visible scattered light produced by a collinear beam from a $\mathrm{He}-\mathrm{Ne}$ laser. The visible scattered light was modulated apparently because of the local photothermally induced change in particle size. When IR radiation is absorbed by a small solution drop in equilibrium with water vapor, the droplet evaporates slightly; it shrinks in size. The evaporation is caused by the increase in vapor pressure at the surface of the drop as its temperature increases. The new smaller size is stable as long as the radiation is continuous. ${ }^{2}$ The mechanism for this phenomenon may be understood for an ideal solution in terms of Raoult's law. Simply stated, the vapor pressure of water at the surface of such a solution is the vapor pressure of pure water $P_{0}$ times the mole fraction of water in the solution $X_{w}$. When a drop is heated, the vapor pressure $P_{0}$ increases; a flux of vapor is emitted into the external medium, and the drop shrinks. Since this shrinkage causes a decrease in the mole fraction of water within the particle, the vapor pressure at the surface of the drop begins to fall. Eventually the particle reaches a vapor pressure equal to the external environment and stabilizes in size.

In the work on a cloud of polydispersed particles, ${ }^{3}$ the size-dependent structure associated with the scattered 


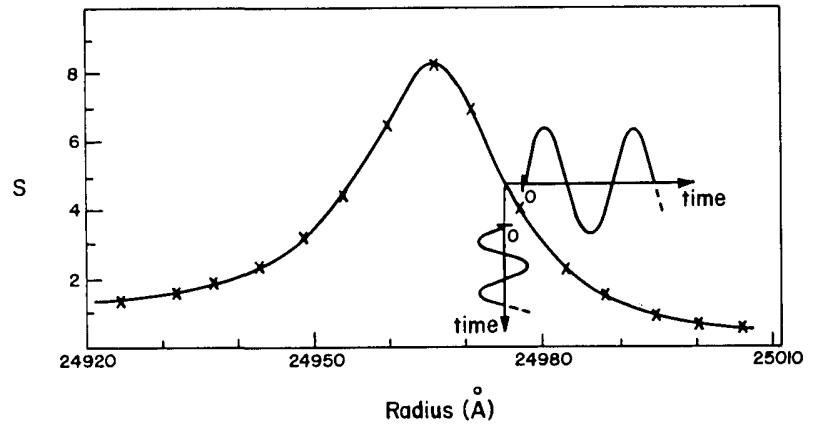

Fig. 1. Back scattered intensity vs particle size for an incident wavelength of $5346 \AA$. The refractive index is 1.331 .

light was neglected, and consequently interpretation of the scattered light intensity was incomplete. This limits the degree to which one can obtain accurate absorption data. In addition the high intensities needed $\left(>1 \mathrm{~W} / \mathrm{cm}^{2}\right)$ in this case limit the spectral range over which measurements can be made. In SRMS these limitations are overcome by using the properties of structure resonances. The chief advantages of this method are a full accounting for size related effects and a reduction in the IR intensity requirements by more than 3 orders of magnitude. ${ }^{1}$ This latter advantage makes possible the use of simple broadband light sources (e.g., Globar) from which a continuous spectrum of the particle may be obtained. ${ }^{2}$

For the weak intensity produced by a monochromatized incandescent IR source $\left(\sim 10-\mathrm{cm}^{-1}\right.$ bandwidth), the size change of a particle $5 \mu \mathrm{m}$ in diameter may be smaller than $1 \AA$. However, this size change is easily detected by utilizing the properties of the particle's structure resonances. To do this the narrowband radiation from a tunable dye laser is positioned at the low-wavelength side of a structure resonance of the particle. This resonance, detected through visible scattered light, ${ }^{4}$ is a natural electromagnetic mode of the particle. ${ }^{5}$ It can be shown ${ }^{1}$ that a fractional change in particle radius $\delta a / a$ leads precisely to the same change in resonant wavelength $\delta \lambda_{r} / \lambda_{r}$. Thus a given resonance is shifted to shorter wavelength as the particle shrinks. If the incident radiation is positioned at the short-wavelength side of a resonance, a reduction in particle size will lead to an increase in scattered light. Figure 1 shows a Mie calculation of the backscattered light $S$ at $5346 \AA$ from a particle with a refractive index of 1.331 in the size range from 2.4920 to $2.5010 \mu \mathrm{m}$. This structure resonance is clear. On the verticle time axis we suppose that the size is made to oscillate harmonically. As a result the scattered light will oscillate as shown on the horizontal time axis. The transfer parameter describing the scattered light change with size is $\beta=(a / S) \cdot(\partial S / \partial a)$. This transfer parameter $\beta$ is obtained by measuring the excitation spectrum of the scattered light near the wavelength of the dye laser. Due to the pronounced nature of these resonances, the excitation spectrum is virtually a function of optical size $(2 \pi a / \lambda)$ near resonance, and as a consequence the wavelength transfer function [i.e. $\left.\left(\lambda_{r} / S\right) \cdot\left(\partial S / \partial \lambda_{r}\right)\right]$ is

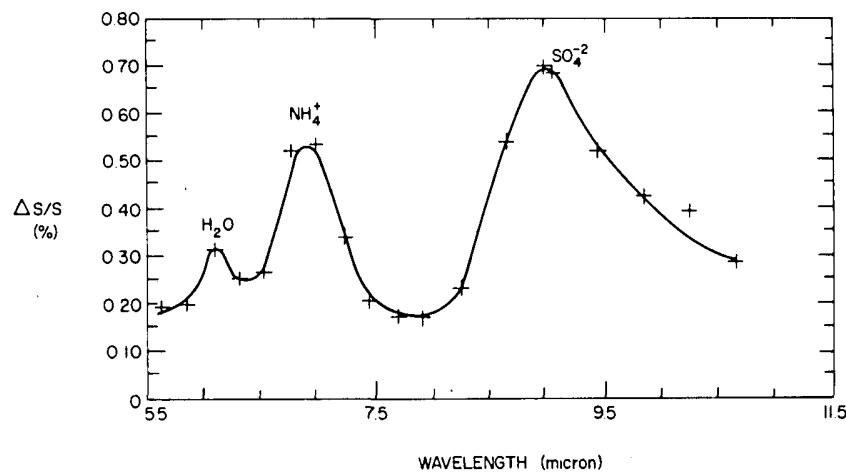

Fig. 2. SRMS spectrum taken on a single solution drop of $\left(\mathrm{NH}_{4}\right)_{2} \mathrm{SO}_{4}$ about $10 \mu \mathrm{m}$ in diameter.

essentially equal to $-\beta$. Thus the excitation spectrum provides us with a direct measure of $\beta$; it calibrates the SRMS technique. The value of $\beta$ at the point of the harmonic disturbance in the backscattering spectrum in Fig. 1 is -2500 . Resonances may also be seen at other scattering angles. In the original work on SRMS by Arnold et $a .^{2}$ the scattered light was observed normal to the incident light. For this experiment a value for $\beta$ of -4300 was measured. For such a value a $-0.1-\AA$ change in radius out of $2.5 \mu \mathrm{m}(4 \mathrm{ppm})$ leads to a $1.2 \%$ change in scattered light. With periodically modulated IR, a fluctuation in scattered light of $\sim 1 \%$ is easily detected.

To obtain an SRMS spectrum we irradiate a levitated microparticle with a temporally chopped IR source and phase sensitively detect the corresponding fluctuation in the visible scattered light. ${ }^{2}$ The IR radiation is provided by a Globar followed by a monochromator (bandwidth $\sim 10 \mathrm{~cm}^{-1}$ ). The visible source is a dye laser (bandwidth $\sim 0.3 \AA$ ) with its radiation tuned to a wavelength in the region of a structure resonance (a wavelength which optimizes the transfer parameter $\beta$ ). The resulting SRMS spectrum is a record of the fluctution in visible scattered light vs IR wavelength.

An example of an SRMS spectrum of an $\left(\mathrm{NH}_{4}\right)_{2} \mathrm{SO}_{4}$ drop is shown in Fig. 2. This drop, which was nearly saturated, was $10 \mu \mathrm{m}$ in diameter. Although the characteristic molecular resonances are broadened by saturation effects due to large particle size in comparison with the absorption depth of the light, the individual vibrational lines for $\mathrm{H}_{2} \mathrm{O}, \mathrm{NH}_{4}^{+}$, and $\mathrm{SO}_{4}^{=}$are evident. To understand both the amplitude and shape of this spectrum one must investigate the physical processes involved. In what follows, we review our current understanding of SRMS, point out its limitations, present a comprehensive model for the spectroscopy, and supply an experimental test of this model.

In earlier work, ${ }^{1}$ we gave an equation relating the fractional change in scattered light $\delta S_{s} / S$ produced by constant IR radiation and measured after the new size equilibrium is reached:

$$
\delta S_{s}\left(\lambda_{2}\right) / S=F_{s} \beta\left(\lambda_{2}\right) Q_{a}\left(\lambda_{1}\right) I\left(\lambda_{1}\right)
$$

where $\lambda_{1}$ and $\lambda_{2}$ are the infrared (excitation) and visible (probe) wavelengths, $Q_{a}$ is the particle absorption ef- 
ficiency, and $I$ is the infrared intensity. With knowledge of the function $F_{s}$, Eq. (1) forms the basis for the measurement of $Q_{a}$ at a constant IR intensity. ${ }^{2}$ The form of $F_{s}$ given in Refs. 1 and 2 applied to the case of a dilute nonelectrolytic solution and is expected to be a crude approximation when applied to concentrated electrolytic solutions. Our first major goal is to arrive at a more realistic form for the factor $F_{s}$.

The static approach to SRMS as described by Eq. (1) is difficult to use in obtaining continuous IR spectra due to the weak intensities available from continuous IR sources. To improve the SNR, it is necessary to use a modulated excitation source and phase sensitively detect the scattered light fluctuations. Consequently, Eq. (1) must be replaced with a dynamical form. Although the dynamical equations governing the coupling between a heated droplet and its vapor field are generally nonlinear, experimental evidence indicates that for the weak intensities used in SRMS $\left(\left\langle 20 \mathrm{~mW} / \mathrm{cm}^{2}\right\rangle\right)$, a simple analytical form can be obtained. Arnold et al. ${ }^{2}$ have shown experimentally that the amplitude of the scattered light modulation $\delta S_{s} \bar{S}$ is directly proportional to $\beta\left(\lambda_{2}\right) \cdot Q\left(\lambda_{1}\right) \cdot I_{1}\left(\lambda_{1}\right)$, where $I_{1}$ is the amplitude of the first harmonic of the IR intensity, and $\bar{S}$ is the average scattered light. Thus we define a new factor $F_{\omega}$ through

$$
\delta S_{\omega}\left(\lambda_{2}\right) / \bar{S}=F_{\omega} \beta\left(\lambda_{2}\right) Q_{a}\left(\lambda_{1}\right) I\left(\lambda_{1}\right) .
$$

In the next section we will provide a physical basis for Eqs. (1) and (2) and obtain expressions for $F_{s}$ and $F_{\omega}$.

\section{Model}

The equations describing the detailed coupling between a heated drop and the associated hydrodynamic modes of external gas and vapor are in general nonlinear. ${ }^{6}$ Due to this inherent nonlinearity, we lean on experimental evidence in developing a model for the interaction of such a drop with weak radiation. Figure 3 shows a typical oscillogram of the visible light scattered from a particle of $\left(\mathrm{NH}_{4}\right)_{2} \mathrm{SO}_{4} 5 \mu \mathrm{m}$ in diameter in the presence of radiation from a Nernst glower. The particle was held in equilibrium at room temperature above a saturated solution of $\mathrm{KCl}$. Addition of $\mathrm{N}_{2}$ gas brought the total pressure to $1 \mathrm{~atm}$. The concentration of $\left(\mathrm{NH}_{4}\right)_{2} \mathrm{SO}_{4}$ in the drop was $38 \%$ by mass. Although the IR intensity is square wave modulated at $20 \mathrm{~Hz}$ one can readily see that the scattered light signal is triangular in form. This different form is not due to a variation in $\beta$ with size. It is rather the result of a phase boundary relaxation process with a characteristic time which is longer than $1 / 40 \mathrm{sec}$, a rate smaller than 40 $\sec ^{-1}$.

The rates usually associated with establishing steady state in vapor and thermal transport, $\beta_{v}$ and $\beta_{t}$, are

$$
\beta_{v} \sim \frac{3 D}{a^{2}} \quad \beta_{t} \sim \frac{K_{g}}{\rho C_{g} a^{2}},
$$

where $D$ is the diffusion constant for water vapor through air, and $K_{g}, \rho$, and $C_{g}$ are the thermal conductivity, density, and specific heat of the gas, and $a$ is the particle radius. For a particle $5 \mu \mathrm{m}$ in diameter in air

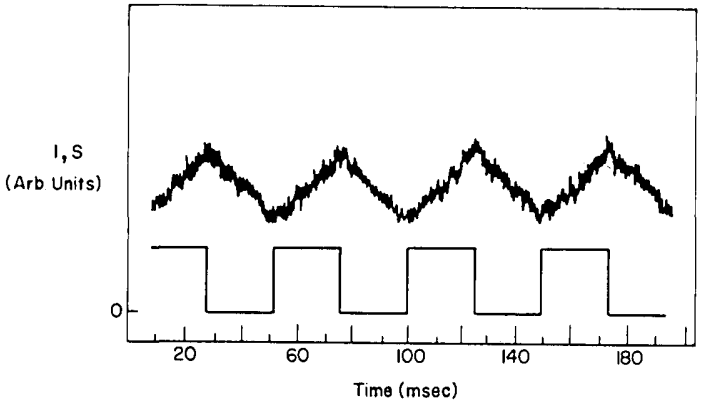

Fig. 3. Oscillogram of both scattered light modulation (above) and incident IR intensity (below) from a $\left(\mathrm{NH}_{4}\right)_{2} \mathrm{SO}_{4}$ particle $5 \mu \mathrm{m}$ in diameter. The frequency of the incident visible light was positioned on the low-wavelength side of a structure resonance.

at atmospheric pressure and room temperature, each of these rates is of the order of $10^{7} \mathrm{sec}^{-1}$. Since the phase boundary relaxation rate estimate from Fig. 3 is $<40 \mathrm{sec}^{-1}$ we will assume that the phenomena described in the previous section may be analyzed with the temperature and vapor fields in steady state. In addition, since the temperature increase over ambient is $<10^{-2 \circ} \mathrm{C}$, hydrodynamic influences on heat and mass transport are expected to be negligible. ${ }^{7}$ With these reasonable assumptions, the temperature and vapor fields will obey Laplace's equation ${ }^{7}$

$$
\nabla^{2} C=\nabla^{2} T=0
$$

outside the particle. The radiative power absorbed by the particle $P_{a}$ is lost through thermal conduction into the ambient gas and through evaporative losses in which each gram lost carries away the appropriate latent heat $L:$

$$
P_{a}=-\oint\left(K_{g} \nabla T+L D \nabla C\right) \cdot \hat{n} d s .
$$

In Eq. (4) the integration is carried out over the surface of the particle. The power absorbed is given properly by Mie theory. ${ }^{8}$ In this theory the power absorbed by the particle is an absorption efficiency $Q_{a}$ times the power incident on the particle's geometrical cross section:

$$
P_{a}=Q_{a} \pi a^{2} I .
$$

Energy absorbed is conducted quickly within the particle so that even though the distribution of internal heat sources is anisotropic, ${ }^{9}$ this anisotropy almost completely vanishes. Consequently we will assume a condition of spherical symmetry. Combining the assumption of steady state [Eq. (3)] with that of spherical symmetry produces vapor and temperature fields:

$$
C(r)=\frac{\left(C_{s}-C_{\infty}\right) a}{r}+C_{\infty} \quad T(r)=\frac{\left(T_{s}-T_{\infty}\right) a}{r}+T_{\infty},
$$

respectively, where $C_{s}$ and $T_{s}$ are the vapor density and temperature at the particle's surface, and $C_{\infty}$ and $T_{\infty}$ are the same quantities far from the particle. Strictly speaking, Eq. (6) should be modified to include the effects of the finite mean free path. In the present case, however, the mean free path is much smaller than the particle size, and the gas is consequently treated as 
continuous; mean free path effects are neglected. In what follows $\left(C_{s}-C_{\infty}\right)$ and $\left(T_{s}-T_{\infty}\right)$ will alternatively be referred to as $\delta C$ and $\delta T$, respectively.

Since the solution will be electrolytic in the present case, the water vapor concentration at the surface is arrived at by utilizing Raoult's law and the van't Hoff factor. ${ }^{10}$ Thus

$$
C_{s}=\left[1-i\left(1-X_{w}\right)\right] C_{p},
$$

where $X_{w}$ is the mole fraction of water in the drop, $C_{p}$ is the vapor concentration above pure water, and $i$ is the van't Hoff factor. Typical perturbations in particle radius for SRMS are small enough ( $<1$ part in $\left.10^{3}\right)$ to allow the analysis of the change in vapor pressure to be carried out to first order in $\delta a / a$. A corresponding approximation will be made for temperature; the analysis will be carried out to first order in $\delta T / T$. On this basis the change in $C_{s}$ due to changes in both $X_{w}$ and $C_{p}$ is

$$
\delta C_{s}=i C_{p} \delta X_{w}+\left[1-i\left(1-X_{w}\right)\right] \delta C_{p} .
$$

The change in $C_{p}$ is obtained by combining the Clausius-Clapeyron equation and the ideal gas law. To first order in $\delta T / T$,

$$
\delta C_{p}=C_{p}\left(\frac{L M}{R T}-1\right) \frac{\delta T}{T},
$$

where $M$ is the molecular weight of the vapor, and $R$ is the universal gas constant. The change in $X_{w}$ is obtained from the change in radius of the particle under the assumption of constant particle density to first order in $\delta a / a$ :

$$
\delta X_{w}=\frac{3 X_{w}\left(1-X_{w}\right)}{f_{w}} \frac{\delta a}{a},
$$

where $f_{w}$ is the mass fraction of water.

Overall $\delta C_{s}$ may be expressed in terms of size and temperature changes by combining Eqs. (8), (9), and (10),

$$
\delta C_{s}=C_{p}\left\{\frac{3 i X_{w}\left(1-X_{w}\right)}{f_{w}} \frac{\delta a}{a}+\left[1-i\left(1-X_{w}\right)\right]\left(\frac{L M}{R T}-1\right) \frac{\delta T}{T}\right\} .
$$

Ultimately we are after the manner in which the radius shrinks with time. A useful relationship for determining this comes from considering the mass loss of the drop. Simply speaking,

$$
\frac{-d m}{d t}=-\oint D \nabla C_{r=a} \cdot \hat{n} d s
$$

On evaluating Eq. (12), using Eqs. (6), we obtain

$$
-\rho_{p} a \dot{a}=D \delta C_{s},
$$

where $\rho_{p}$, the particle density, is assumed to remain constant. Recall that experimentally we are looking at a change in radius of $<1$ part in $10^{3}$. Consequently, the radius will be considered to be a sum of a constant part $a_{0}$ and a time varying part $\varepsilon(t)$. Combining Eqs. (13) and (11) we obțain

$$
-\dot{\epsilon}=\frac{D C_{p}}{\rho_{p} a_{0}}\left\{\left[1-i\left(1-X_{w}\right)\right]\left(\frac{L M}{R T}-1\right) \frac{\delta T}{T}+\frac{3 i X_{w}\left(1-X_{w}\right)}{f_{w}} \frac{\varepsilon}{a_{0}}\right\} .
$$

This simple linear first-order differential equation [Eq.
(14)] is almost our equation of motion. However, we must express $\delta T$ in terms of the incident intensity and other relevant physical quantities. To do this we return to Eq. (4). Evaluating Eq. (4) [using Eqs. (5) and (6)] we obtain

$$
\delta T=\frac{1}{K_{g}}\left(\frac{Q_{a}}{4} I a-L D \delta C_{s}\right) .
$$

Substituting for $\delta C_{s}$ in Eq. (15) from Eq. (11), we obtain

$$
\delta T(1+L Z)=\frac{1}{K_{g}}\left[\frac{Q_{a} I a}{4}-\frac{3 i L D C_{p} X_{w}\left(1-X_{w}\right)}{f_{w}} \frac{\varepsilon}{a_{0}}\right],
$$

where

$$
Z=\frac{D C_{p}}{K_{g} T}\left[1-i\left(1-X_{w}\right)\right]\left(\frac{L M}{R T}-1\right) .
$$

Finally, by combining Eq. (16) with Eq. (14) we obtain a linearized equation for the motion of the phase boundary:

$$
\dot{\epsilon}=-\alpha I-\gamma \varepsilon,
$$

where

$$
\begin{aligned}
& \alpha=\left(\frac{Z}{L Z+1}\right) \frac{Q_{a}}{4 \rho_{p}} \\
& \gamma=\frac{3 D C_{p} i}{\rho_{p} a_{0}^{2} f_{w}} \cdot \frac{X_{w}\left(1-X_{w}\right)}{L Z+1}+\frac{Q_{a} I Z}{4 a_{0} \rho_{p}(L Z+1)} .
\end{aligned}
$$

For the intensities normally used in SRMS the second term in Eq. (18) is much smaller than the first and may be neglected. For example, in the first experiments by Arnold $e t a l .^{2}$ one calculates that the first term in Eq. (18) is more than 100,000 times the second term. Thus the relaxation rate $\gamma$ in our model is, to within a good approximation, independent of the absorbed energy. This is distinct from the case of pure water (i.e., $1-X_{w}$ $=0$ ), where the relaxation rate is directly proportional to the absorbed energy, and any imbalance in temperature leads to complete evaporation. ${ }^{11}$ It is interesting to compare $\gamma$ with the vapor relaxation rate $\beta_{v}$. Using Eqs. (1) and (18) we find

$$
\gamma=\beta_{v}\left(\frac{i C_{p}}{\rho_{p} f_{w}}\right) \cdot \frac{X_{w}\left(1-X_{w}\right)}{L Z+1} .
$$

Since the ratio of the density of water vapor to the density of liquid water, $C_{p} / \rho_{p}$, near room temperature is $10^{-5}$, one immediately can see the large effect which evaporation has on the relative magnitude of $\gamma$ and $\beta_{v}$. The factor $X_{w}\left(1-X_{w}\right) /[L Z(L)+1]$ further lowers $\gamma$ with respect to $\beta_{v}$. For the conditions for which the scattered light fluctuations were recorded in Fig. 3 we calculate $\gamma$ to be $11.6 \mathrm{sec}^{-1}$. Consequently the phase boundary would take $86 \mathrm{msec}$ to relax, which is consistent with the lower limit estimated from Fig. 3. In what follows we will attempt to test Eq. (19) in a more quantitative fashion.

\section{Experimental}

Here we describe the experimental setup and procedure used in measuring the relaxation rate $\gamma$. The experimental value of $\gamma$ obtained here is a direct test of the theoretical development in the previous section. 


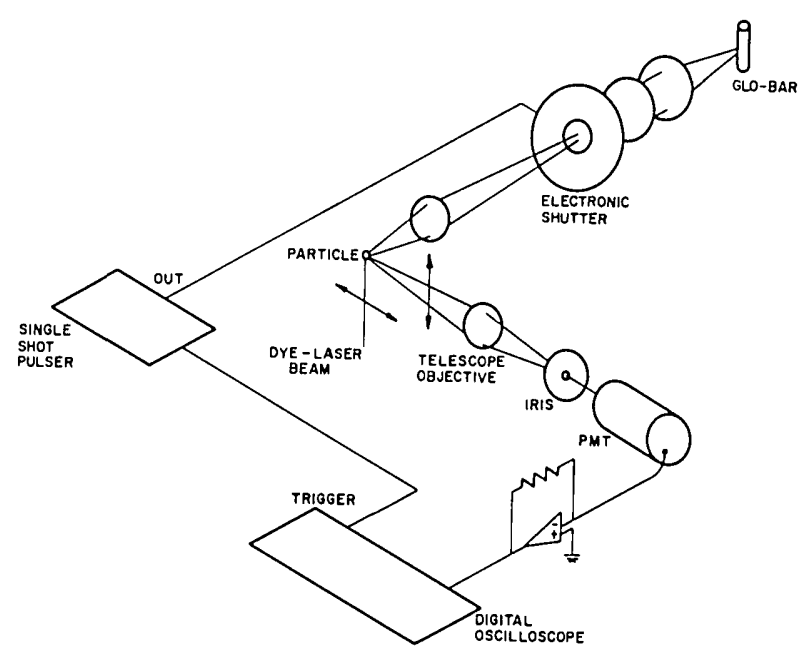

Fig. 4. Experimental setup for transient light scattering measurements.

The particle is electrically suspended within a sealed chamber by use of an electrodynamic trap. ${ }^{12}$ This trap is optically servo controlled ${ }^{13}$ so that the particle is maintained at the center of the chamber, and its mass is directly monitored. A sample particle was prepared by injecting a dilute solution droplet of ammonium sulfate from an impulse jet into the chamber. Once the drop was trapped, the chamber was evacuated down to a pressure of 1 Torr, at which point the particle consisted of solid ammonium sulfate.

After the evacuation, water vapor was bled into the chamber from a tube attached to the vacuum manifold. The relative humidity in the chamber was maintained at a constant level by utilizing a saturated salt solution as the source of water vapor. Since the relative humidity in the chamber exceeded the deliquescence point of $\left(\mathrm{NH}_{4}\right)_{2} \mathrm{SO}_{4}$, the particle absorbed water. The deliquescence point was observed optically by the gradual appearance of well-defined Mie patterns in the far field. Additionally, the increase in weight of the particle was observed by the increase in balancing voltage. At size equilibrium, the mass fraction of water in the particle was obtained from the levitating voltage before and after the addition of the vapor. At this point $\mathrm{N}_{2}$ was bled into the chamber so that the experiment was run at a total pressure of 200 Torr. The pressure was raised to decrease the mean free path of the gas molecules so that heat and mass transport processes would be in the realm of low Knudsen numbers, the continuum regime, consistent with our proposed model. After the desired pressure was reached, the positions of a few structure resonances were detected in the frequency spectrum of Mie scattering 4 at $90^{\circ}$. From the measured positions of these resonances, which were found to be stable (consistent with vapor pressure equilibrium), the size of the particle was determined. ${ }^{5}$ At this point the sample was irradiated with a square pulse of broadband radiation from a Nernst glower (Fig. 4). The pulse was generated for a specific time by utilizing an electromechanical shutter with opening and closing times of 1 msec.

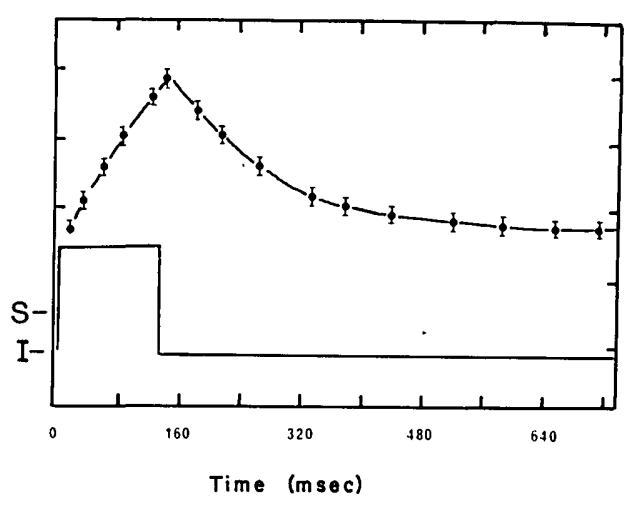

Fig. 5. Light scattering transient from a solution particle of $\left(\mathrm{NH}_{4}\right)_{2} \mathrm{SO}_{4}$ with a radius of $6.2 \mu \mathrm{m}$ and a water mole fraction of 0.89 \pm 0.01 . The lower trace shows the measured IR pulse.

For short heating periods and fast relaxation rates the response of the particle to the heat pulse was obtained from the change in the scattered light. In this case, the dye laser was tuned to the low side of a structure resonance where a small fluctuation in the particle size causes a large fluctuation in the scattered light. For longer heating periods and relaxation rates the particle response was observed directly from the changes in the balancing voltage. In all cases, the response of the particle was recorded on a digital oscilloscope. Data from such a recording are shown in Fig. 5. As one can see, the particle relaxed back to its original size once the heat source is turned off.

For the data shown in Fig. 5, the particle size as measured from the position of structure resonances ${ }^{5}$ was $6.2 \mu \mathrm{m}$. The mole fraction of water in this particle was measured to be $0.89 \pm 0.01$, consistent with complete saturation at $25^{\circ} \mathrm{C} .{ }^{14}$ From the ambient temperature and associated thermodynamic constants in Eq. (18), we calculate a relaxation rate of $173 \mathrm{msec}$ in good agreement with the measured value of $160 \pm 20 \mathrm{msec}$ taken from the logarithmic fit shown in Fig. 6. The

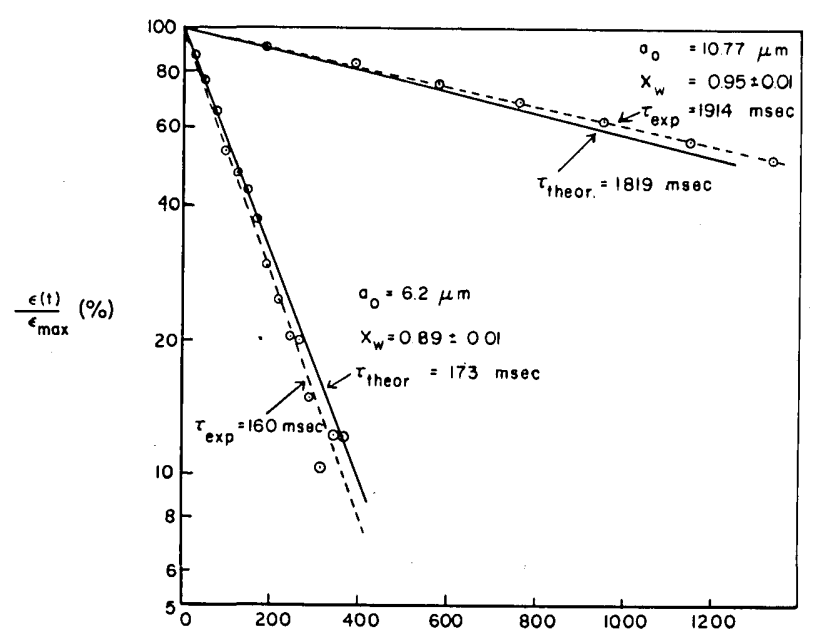

Fig. 6. Scattered light vs time after IR intensity is turned off. The solid lines are theoretical predictions based on Eq. (19). The pressure of $\mathrm{N}_{2}$ gas plus vapor was 200 Torr. 
solid lines in Fig. 6 are theoretical predictions based on Eq. (17). From the other experiment in Fig. 6 we observe that a larger particle containing more water has a smaller relaxation rate, consistent with theory.

\section{v. Discussion}

Based on the good agreement between our model and the experimental results shown in Fig. 6, we use the equations developed earlier to calculate the new functionality of the factor $F$ in Eq. (1). For constant excitation, $\dot{\epsilon}=0$, Eq. (17) gives the particle size change at equilibrium as

$$
\varepsilon_{e}=-\frac{I \alpha}{\gamma} .
$$

Recalling that $\delta S / S=\beta_{\varepsilon} / a_{0}$, one can substitute Eqs. (18) and (20) into Eq. (1) and obtain the following expression for $F_{s}$ :

$$
F_{s}=\frac{Z a_{0} f_{w}}{12 D C_{p} i X_{w}\left(1-X_{w}\right)} .
$$

It should be pointed out that Eq. (21) reduces to the earlier expression presented in Refs. 1 and 2 in the limit of dilute nonelectrolytic solutions.

For a periodically modulated excitation source, at angular frequency $\omega$, the factor $F$ in general will depend on $\omega$. If we suppose a harmonic excitation $I=I_{1}$ $\exp (j \omega t)$, the corresponding size fluctuation is

$$
\varepsilon_{1}(t)=-\frac{\alpha I_{1}}{\left(\omega^{2}+\gamma^{2}\right)^{1 / 2}} \exp [j(\omega t+\phi)]
$$

where $\phi=\tan ^{-1}(-\omega / \gamma)$. In practice the quantity $I_{1}$ is the first Fourier coefficient of the modulated intensity (e.g., $I_{1}=2 I_{m} / \pi$ for a square wave, where $I_{m}$ is the maximum intensity). Thus in terms of the amplitude of the first harmonic of the scattering signal (as detected by a phase sensitive detector), we find the value of $F_{\omega}$ to be

$$
F_{\omega}=\frac{Z}{4 a_{0} \rho_{p}\left(\omega^{2}+\gamma^{2}\right)^{1 / 2}(L Z+1)} .
$$

From Eq. (23) one sees the large effect of the modulation frequency $\omega$ on the function $F_{\omega}$ and, therefore, on the scattering light fluctuation. For large particles, where $\gamma$ may be $<1 \mathrm{sec}^{-1}$, one must go to extremely slow modulations [linear frequency $<(2 \pi)^{-1} \mathrm{~Hz}$ ] to optimize the signal. Fortunately, for aerosol size particles near deliquescence, more manageable values for $\gamma$ are obtained.

\section{Conclusions}

In the foregoing, we have presented a simple model for understanding the kinetics of the processes accompanying the structure resonance modulation of a solution droplet. Our experiments indicate that the model contains most of the essential features which are involved. Our assumptions are specific in applying to the case of low intensity and low Knudsen number. The sensitivity of the relaxation rate to size suggests that our model will prove useful in determining the degree of polydispersivity in experiments on the temporal behavior of the photothermal modulation signal from aerosols. ${ }^{3}$ Certainly as one reduces ambient pressure further, the importance of finite mean free path will complicate further our model. Wagner ${ }^{15}$ has extensively investigated evaporation and condensation in quasi-thermal-equilibrium and finds that the efficiency for water sticking enters both transfer processes as the Knudsen number increases. On this basis one may expect that phase boundary relaxation measurements made in the large Knudsen number regime may be used to investigate water sticking. Such an investigation is currently under way in our laboratory.

We would like to thank M. Neuman (presently at the National Research Council, Manitoba) for taking some preliminary data in this study. We are grateful to Jeff Rosenfeld of the Microparticle Photophysics Laboratory (at the Polytechnic) for his help in computer interfacing. One of us (E. K. M.) was cooperatively supported by a grant from The National Science Foundation and the Chemical Research and Development Center of the Army under NSF grant ATM-8413574. S. Arnold was also supported by this grant and a U.S. Joint Services Electronics contract (F49620-82-C0084).

\section{References}

1. S. Arnold and A. B. Pluchino, "Infrared Spectrum of a Single Aerosol Particle by Photothermal Modulation of Structure Resonances," Appl. Opt. 21, 4194 (1982).

2. S. Arnold, M. Neuman, and A. B. Pluchino, "Molecular Spectroscopy of a Single Aerosol Particle," Opt. Lett. 9, 4 (1984).

3. A. J. Campillo, C. J. Dodge, and H.-B. Lin, "Aerosol Particle Absorption Spectroscopy by Photothermal Modulation of Mie Scattered Light," Appl. Opt. 20, 3100 (1981).

4. A. Ashkin and J. M. Dziedzic, "Observation of Optical Resonances of Dielectric Spheres by Light Scattering," Appl. Opt. 20, 1803 (1981).

5. P. Chylek, J. T. Kiehl, and M. K. W. Ko, "Optical Levitation and Partial Wave Resonances”, Phys. Rev. A 18, 2229 (1978).

6. G. Sageev and J. H. Seinfeld, "Laser Heating of Aqueous Aerosol Particles" Appl. Opt. 23, 4368 (1984).

7. L. D. Landau and E. M. Lifshitz, Fluid Dynamics (Pergamon, London, 1959).

8. G. Mie, "Beitrage zur Optik tüber Medien Speziell Kolloidaler Metallösungen," Ann. Phys. 25, 377 (1908).

9. P. W. Dusel, M. Kerker, and D. D. Cooke, "Distribution of Absorption Centers Within Irradiated Spheres," J. Opt. Soc. Am. 69, 55 (1979); A. B. Pluchino, "Photophoretic Force on Particles for Low Knudsen Number," Appl. Opt. 22, 103 (1983); S. Arnold and M. Lewittes, "Size Dependence of the Photophoretic Force," J. Appl. Phys. 53, 5314 (1982).

10. See, for example, E. A. Moelwyn-Hughes, Physical Chemistry (Pergamon, London, 1957), p. 823.

11. M. B. Baker, "Energy Absorption by Volatile Aerosol Particles," Atmos. Environ. 10, 241 (1976).

12. M. A. Philip, F. Gelbard, and S. Arnold, "An Absolute Method for Single Aerosol Particle Mass Measurement," J. Colloid Interface Sci. 91, 507 (1982).

13. S. Arnold, Y. Amani, and A. Orenstein, "Photophoretic Spectrometer," Rev. Sci. Instrum. 51, 1202 (1980).

14. B. F. Wishaw and R. H. Stokes, "Activities of Aqueous Ammonium Sulphate Solutions at $25^{\circ} \mathrm{C}$," Trans. Faraday Soc. 50, 952 (1954).

15. P. E. Wagner, "Aerosol Growth by Condensation," in Aerosol Microphysics II W. H. Marlow, Ed. (Springer, Berlin, 1982), Chap. 5, pp. 129-178. 